\title{
A Study on the Formation and Evolution Model of Social Psychology in Major Public Health Emergencies
}

\author{
Chenhe $\mathrm{Yi}^{1}$, Shan $\mathrm{Li}^{1 *}$ \\ ${ }^{1}$ School of Public Administration, Xiangtan University Xiangtan, Hunan P.R. China
}

\begin{abstract}
The occurrence of local or regional major public health emergencies has seriously damaged human health and life safety, and has an impact on the psychological state of the public. The study introduces"social amplification of risk"effect and the mechanism of emotional infection, and constructs a process model of psychological prediction, psychological cognition and psychological behavior in the generation of social psychology of major public health emergencies, and from the level of individual emotions, group emotions and social emotions analyze the evolutionary model of social psychology; Research has found that information disclosure, government trust and social confidence are the main factors influencing the formation of social psychology of major public health emergencies.
\end{abstract}

\section{Introduction and Literature Review}

Major public health emergencies refer to major infectious disease outbreaks, mass diseases of unknown causes, major food and occupational poisonings, and other events that seriously affect public health that occur suddenly and cause or may cause serious damage to public health [1]. It usually occurs suddenly without human beings' knowledge, making it difficult to prevent, and has the characteristics of crisis events such as complex diversity, widespread transmission, and severity of harm. It generally affects and changing the mental structure and mental activities of social members, causing the public to produce mental states such as panic, anxiety, and questioning, and even forming lingering psychological shadows and psychological behaviors, which may spread and continue to sudden public health emergencies daily life and behavior after the event. Based on this, the social psychological impact and intervention work in public emergencies has gradually attracted the attention of the Chinese government. For example, government departments have clearly stipulated the level of social mental health services from the perspective of laws and regulations[2-4]. However, in fact, there are many difficulties in the intervention and regulation of social psychology in major public health emergencies, especially in the modern complex social environment, the speed of information dissemination has accelerated the production and change of social psychology in public health emergencies. Therefore, grasping the general laws of the generation and evolution of social psychology in public health emergencies will help the government to correctly understand and scientifically guide the nature and trend of the generation and evolution of social psychology.
Compared with the influence of social politics, economic industry and public security, the impact of major public health emergencies on social value and psychological state is relatively unique and hidden, and it has attracted more and more researchers' attention. First of all, the existing research on the evolution of social psychology is mostly analyzed from the cognitive level or the perceived value dimension. For example, Some scholars have found that social psychology is the general public perception and cognition of social reality in a specific environment or a specific event.[5-7]Some scholars think in public health emergencies, social psychology usually resonates at the same frequency as online public opinion and group behaviors, and has an amplifying effect under the influence of risk perception bias and emotional infection mechanisms [8-9].Related research shows that there is a universal logical relationship between major public health emergencies and social psychology. Many behavioral factors such as information disclosure, response measures, prevention and control methods will affect the occurrence and dissemination of major public health emergencies. The social psychological state, emotional expression and behavior patterns constitute the influence. This mechanism of action and influence includes not only short-term effects such as physiological emotions and emotional tone, but also longterm effects such as value orientation, behavior habits, and social customs. Therefore, it is extremely necessary to study the social psychology and mentality adjustment in response to major public health emergencies, especially the analysis of the social psychology generation and evolution mechanism of major public health emergencies. It will have important guiding significance for the adjustment of social mentality and the reshaping of social values in response to public health emergencies. Therefore, one of the goals of this research is to analyze the main 
influencing factors of social psychology in major public health emergencies and obtain the relationship between public risk perception and coping behaviors and psychological changes in emergencies, in order to help the public and the government to form an accurate understanding of social psychology. another goal of the research is to build a model of the generation process and evolutionary situation of social psychology in major public health emergencies through the understanding and grasp of social psychology.

\section{Research design}

\subsection{Research method}

The specific research method can be summarized as the five processes of "determining the research problem determining the appropriate text overall-text content analysis and classification-selecting representative textsobtaining qualitative results "[10-11].First of all, because this research is an interdisciplinary basic research, it is necessary to use the basis of text content study to scientifically screen the overall text, and the original text is generally based on "public health emergencies", "social psychology in public emergencies", and "crisis scenarios" "Social Psychology",etc.,conduct preliminary grouping, eliminate invalid and duplicate data, and extract and refine representative texts that are highly consistent or highly related to research content and research purposes; secondly, the extracted representative texts are classified according to the logical relationship between the categories, and the text content can be formed for comparative research, which can be used to sort out and analyze the social psychology generation process and evolutionary context of major public health emergencies.

\subsection{Research steps}

This research focuses on exploring the general laws of the generation and evolution of social psychology in major public health emergencies, and constructs a theoretical model of the generation and evolution of social psychology in major public health emergencies, in an attempt to provide the government with a correct understanding of social psychology. And then provide reference suggestions for determining social psychological early warning and intervention indicators, establishing and continuously improving the early warning system of social psychological behavior indicators. Specifically, the first step is to determine the main influencing factors of the generation of social psychology based on the logical relationship and change characteristics between the subject and the object of social psychology in major public health emergencies; the second step is to determine the main influencing factors of social psychology. Importance, combined with risk management theory and "cognition-evaluation" theory, etc., to construct a model of the generation and evolution of social psychology in major public health emergencies.

\section{Theoretical model construction}

\subsection{Socio-psychological influence factors}

Generally speaking, social psychology is the general public's perception and understanding of social phenomena and social activities. It is the core demand and intrinsic motivation for stimulating and regulating public behaviors for the opportunities, challenges and threats faced in social life [12]. In response to major public health emergencies, social psychology is different from the usual characteristics of social psychological changes, and is more susceptible to developments. It mainly stems from the public's information release, strategic decisionmaking and society in the prevention and control of major public health emergencies. First, information disclosure. American scholar Shannon pointed out that "information is used to eliminate random uncertainties" [13-14] Especially in major public health emergencies, information determines the public's cognitive level and psychological judgment of public health emergencies. Practice has proved that people are always full of distance with unfamiliar emergencies, and they are used to selfdigesting and understanding with guessing, doubting, and even negative psychology. In this case, if the official government information is not released in the first time, it will be eliminated. The subject's one-sided cognition of the object's information will flood the society with relevant false information and rumors, leading to social suspicion, panic, confrontation and other cognitive biases and behaviors, which will seriously affect the correct social psychology in response to major public health emergencies. form. Second, government trust. From a psychological point of view, trust is an individual's perception, judgment and subjective attitude towards outsiders and things, and its essence is interpersonal trust. As an extended type of interpersonal trust, government trust is the core content of the concept of social influence, especially when the public interacts with the government, when facing external crises or risks, they still remain positive about the reliance and reliability of government intentions or administrative actions look forward [15]. In fact, the public's trust in the government largely affects social psychology and behavior. Different groups only have differences in the degree of trust in the government, and there is no difference between them. During the special period of major public health emergencies, in the face of sudden social risks, the public will show different levels of trust in the government's administrative capabilities and expected effects in the prevention and control of public health emergencies. Third, social confidence. Confidence can neither be produced independently nor can it exist independently. It is the result of human beings' positive expectations for a specific behavior or the development and evolution of a certain thing. It is a subjective judgment of future expectations based on realistic cognition. In response to major public health emergencies, public confidence refers to the optimistic evaluation of the public based on the social system's cognition and grasp of the state and evolution of the event, as well as the trust in the social investment 
power of event governance, including individuals. The confidence index also includes the group confidence index.On the contrary, it will form negative collective consciousness and cognitive savings, and lead to social structural resentment [16].

\subsection{Social psychology generation model}

The social psychology of major public health emergencies mainly comes from the public's psychological judgment on the risk or potential risk of public health emergencies and the existing risk prevention and control technology. Constructing a theoretical model of the generation of social psychology in major public health emergencies can link major public health emergencies with risk assessment, risk awareness, risk perception, etc., and analyze the logical relationship between the public's psychological judgments and decision-making behaviors. variable. The subjective risk of major public health emergencies is the public's subjective judgment and prediction of the potential harm of public health emergencies. It will follow the disclosure of prevention and control information and technical upgrades, as well as the public's psychological prediction and psychological recognition of the risk Knowledge and psychological behaviors show a partial normal distribution curve trend. According to the trend of risk perception curve and the analysis framework of "risk social amplification" [17], can represent the process model of social psychology in major public health emergencies as shown in Figure 1.

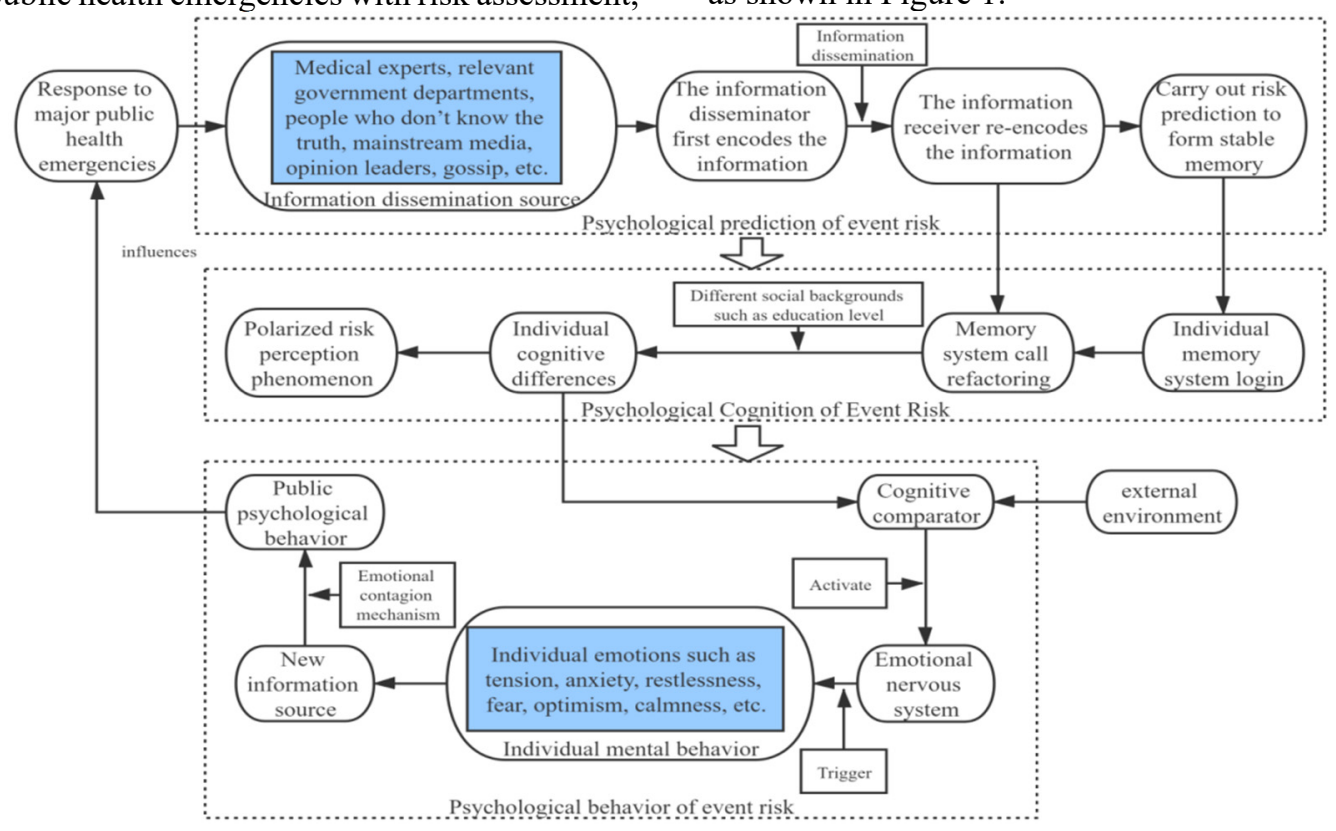

Figure 1: Schematic diagram of the process model of social psychology in major public health emergencies

The model includes three generation stages of psychological change process: First, the psychological prediction of event risk. In the early stage of major public health emergencies, especially when a new or rare public health event is involved, due to the uncertainty of the source of information dissemination, information transmitter will receive different information according to their own knowledge level and society. Encode information on experience, emotional experience, etc., and publish or publicize the processed information through the Internet, self-media, etc. As the receivers of information and the direct associates of event risks, the general public will, in the absence of relevant professional knowledge and reliable truth information, form psychological predictions and directional thinking inertia for event risks based on the relevant information they have obtained. Second, the psychological perception of event risk. The psychological cognition stage of the risk of public health emergencies mainly includes cognitive steps such as individual memory system login, memory system recall and reconstruction, and risk assessment.[18] During the occurrence of major public health emergencies, people's awareness of the risk of the event will be based on the individual's knowledge level, values, and professional differences, as well as the development of different states of the event, mainstream media information reporting, and incident prevention strategies. Third, the psychological behavior of event risk. Psychological cognition reflected in the result is the implementation of social psychological behavior. As far as the individual is concerned, after the second stage of psychological cognition, an optimistic or pessimistic psychological cognition of the risk of the event will be formed, and then through the cognitive comparator in the human brain system, the emotional nervous system will be activated to trigger the implementation of individual psychological behaviors.

Take the new crown pneumonia outbreak that broke out in early 2020 as an example. After the outbreak, the Social Psychology Research Center of the Institute of Sociology of the Chinese Academy of Social Sciences conducted two rounds of investigations on social psychology during the epidemic (the first round: 16: January 24, 2020 16: 40_January 25, 2020 12:50; Second round: January 25, 2020 12:51 — January 29, 2020 $12: 50),[19]$ the survey comparison results are shown in Figure 2: 


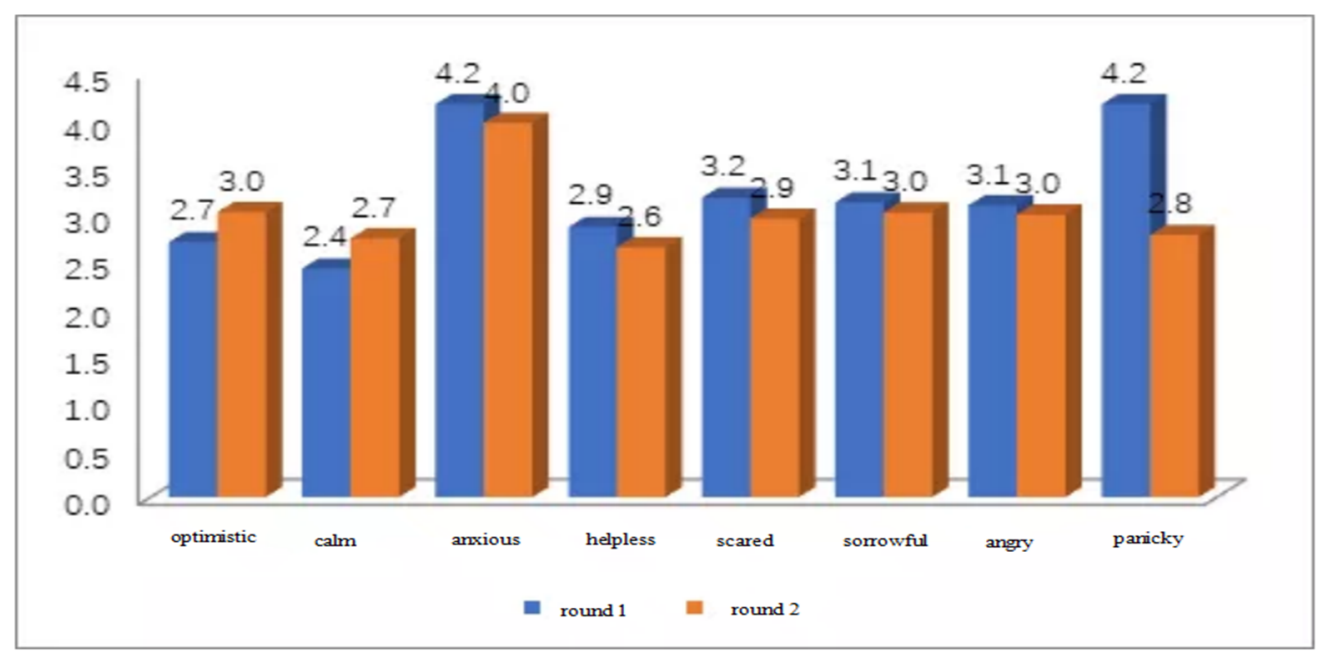

Figure 2: Comparison of social and psychological changes during the new crown epidemic

Data source: Social Psychology Research Center, Institute of Sociology, Chinese Academy of Social Sciences

As shown in Figure 2, the public forms different risk memories after psychologically predicting event risks. At this stage, social psychology is dominated by negative emotions such as worry, helplessness, and fear; however, with the development of events and government risk prevention. With the upgrading of control technology, the public has developed new psychological perceptions of events, and the negative memory storage in the brain has also transformed into positive emotions such as "government trust" and "social confidence". Accordingly, the public's positive social psychological behavior began to rise. As shown in the figure above, although the difference between the two rounds of surveys is only one day apart, the average scores of the public's "optimism" and "calm" mentality have both increased by 0.3 points, while negative mentalities such as "helpless" and "panic" have varying degrees decline, especially the average score of "panic" mood dropped by 1.4 points.

\subsection{Social psychological evolution model}

Through the above analysis, it is found that the generation and change of social psychology and its external performance characteristics in major public health emergencies reflect the role of emotion in the formation and evolution of social psychology. Therefore, when studying and analyzing the generation and evolution mechanism of social psychology, it can be assumed that the basic condition of social psychology is the concentration of social public emotions, and the activation of public emotions is the basic path and way of social psychology evolution, individual psychological emotions form a more common collective psychological cognitive state and behavior through other individual emotional effects (infection, transmission, co-op, imitating, etc.), which evolve into the public's social psychological state. The specific evolution mechanism model is shown in Figure 3.

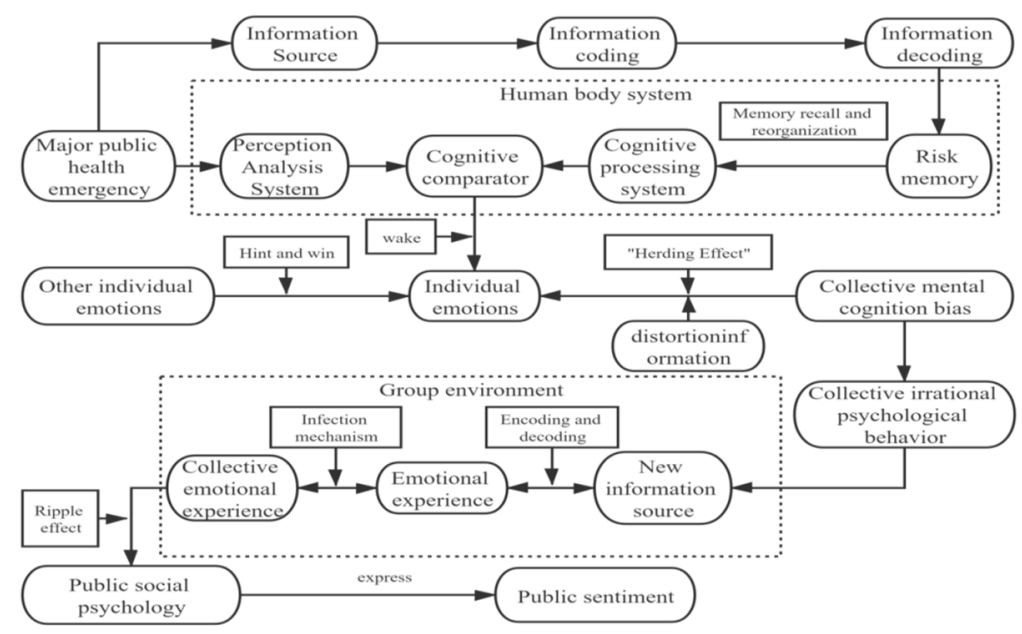

Figure 3: Schematic diagram of the social and psychological evolution mechanism in major public health emergencies

First, Individual emotions. According to Lindsay and Norman, an emotional arousal model was established to explain the generation of emotions under the cognitive processing system [20]. Individuals first encode and decode information about public health emergencies and related phenomena to complete cognitive judgments. If 
the perception analysis system feels the external environment and the conclusions drawn from the analysis of the cognitive processing system are sufficiently consistent, the event will develop steadily and will not generate individual emotions. But due to the contingency and complexity of major public health emergencies, it is different from individual risk memories, usually, the occurrence of events will exceed expectations, predictions, or even violate individual experience, memory and expectations, thereby stimulating the neural activation system to change the state of brain activity and awaken individual emotions. Second, Group emotions. The "herd effect" emphasizes that a single individual can easily lead to a herd mentality when it is guided or pressured by an external group, and therefore changes its original views, judgments and behaviors to maintain an attitude consistent with most other individual members. Third, Social emotions. In the case of a major public health emergency, the public cannot obtain accurate information about the event, resulting in information cascade and information distortion, and collective psychological cognitive bias, this cognitive bias is the main cause of the irrational psychological behavior of collectivity.As some researchers have pointed out, in the process of emotional agglomeration, the original emotions will be further presented in the public opinion field through new stimulus factors and deepen the collective emotional agglomeration of the group [21] and eventually, social psychology is generated and externalized into social emotions. For example, during the new crown pneumonia epidemic (January 24, 2020 to February 10, 2020), with the changes in the degree of publicity of the epidemic and related information, the public's social sentiment has also shown a trend of constant fluctuations, [22] as shown in Figure 4 show.

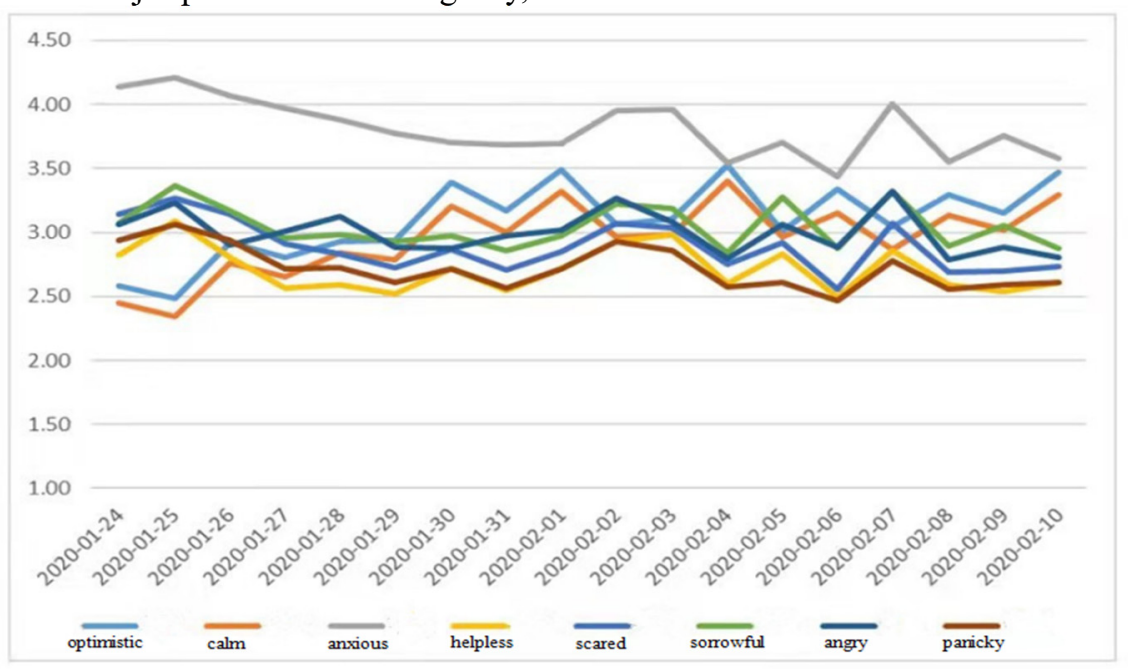

Figure 4: Trends in public social sentiment during the COVID-19 outbreak (2020.1.24-2020.2.10)

Data source: Social Psychology Research Center, Institute of Sociology, Chinese Academy of Social Sciences

\section{Conclusion}

By applying the theory of emotional contagion to group scenarios, combining the characteristics of major public health emergencies and social psychological changes, the study found that information disclosure, social trust and public confidence are the core influencing factors in the formation of social psychology; and on this basis, the theory of "risk social amplification" and "emotional arousal model" are used as intermediary variables to explore the general laws of the generation and change of social psychology, and construct a theoretical model of the generation and evolution of social psychology.The research conclusions drawn mainly include the following two aspects: 1) The generation process of social psychology in major public health emergencies goes through three stages of "psychological prediction of event risk-psychological cognition of event risk-psychological behavior of event risk". In this process, relevant information sources, information dissemination mechanisms, emotional infection mechanisms, and individual subjective factors of major public health emergencies all play an important role. 2) The evolutionary mechanism of social psychology in major public health emergencies is a continuous process of "infection" and transmission, which will respectively present three general social psychological states of individual emotion, group emotion and social emotion. Specifically, a single individual generates individual emotions through the cognitive system under the stimulation of external information sources; individual emotions in the public opinion field through the mobilization mechanism and the infection mechanism, produce collective psychological cognitive bias; finally, through the ripple effect and The linkage mechanism realizes collective irrational behavior in the group environment, which evolves into the public's social psychological state.

\section{Acknowledgment}

This research is supported by the National Social Science Foundation of China (No.16BGL177) 


\section{References}

1. Feng Zhang. Research on risk management of major public health emergencies based on big data. Theoretical Vision, (09):67-73 (2020).

2. The General Office of the State Council of the People's Republic of China. Notice on Further Strengthening Guidance Opinions on Mental Health Work[EB/OL].http://www.gov.cn/xxgk/pub/govpubli c/mrlm/200803/t20080328_32404.html.

3. The website of the Central People's Government of the People's Republic of China. The Mental Health Law of the People's Republic of China[EB/OL].http://www.gov.cn/jrzg/201210/26/content_2252122.htm.

4. The website of the Central People's Government of the People's Republic of China 22 departments issued the "Guiding Opinions on Strengthening Mental Health

Services"[EB/OL].http://www.gov.cn/xinwen/201701/24/content_5162861. htm\#1.

5. Tingqian Zhang. The ethical form and generation logic of the qualitative differentiation of social mentality. Jianghan Forum,(02): 41-49 (2021).

6. Xiaomi Zhou.A review of social psychological analysis and research in mass incidents. Reform and Opening, (02):84-85+89 (2018).

7. Binyi Zhang.Social and psychological guidance inpublic health emergencies.Cities and Disaster Reduction,2021,(01):5-8.

8. Sohail, M. T., Mahfooz, Y., Azam, K., Yen, Y., Genfu, L., \& Fahad, S. Impacts of urbanization and land cover dynamics on underground water in Islamabad, Pakistan. Des, and water treat, 159, 402-411(2019).

9. Sohail, M. T., Mahfooz, Y., Aftab, R., Yen, Y., Talib, M. A., \& Rasool, A. Water quality and health risk of public drinking water sources: a study of filtration plants installed in Rawalpindi and Islamabad, Pakistan. Des. and water treat., 181, 239-250 (2020).

10. Xiaoping Jiang,Ning Guo.The policy goals and evolutionary laws of the equalization of basic publicservices in my country-Based on the textual analysis of the central policy since the 18th National Congress of the Communist Party of China. Public Management and Policy Review,40(06):33-42 (2020).

11. Sohail, M.T., Ullah, S., Majeed, M.T. Pakistan management of green transportation and environmental pollution: a nonlinear ARDL analysis. Environl Sci and Poll Res, 1-10, (2021).

12. Xuefeng Chen, et al.Basic social motivation and social psychological service system construction[J].Advances in Psychological Science,2020,(01):13-21

13. Liangrong Li,Yuqiao Zhou.Constructing the academic discourse system of journalism in continuous exploration[J].Journalism
14. General Office of the State Council of the People's Republic of China. Regulations of the People's Republic of China on Disclosure of Government Information[EB/OL].http://www.gov.cn/zhengce/con tent/2019-04/15/content_5382991.htm.

15. Sohail, M. T., Delin, H., Talib, M. A., Xiaoqing, X., \& Akhtar, M. M. An analysis of environmental law in Pakistan-policy and conditions of implementation. Research Journal of Applied Sciences, Engineering and Technology, 8(5), 644-653 (2014).

16. Li Zhu.A Social Psychological Interpretation of the Gathering and Scattering of Public Confidence. People'sForum, (05):11 (2013).

17. Yen, Y., Wang, Z., Shi, Y., Xu, F., Soeung, B., Sohail, M. T., ... \& Juma, S. A. The predictors of the behavioral intention to the use of urban green spaces: The perspectives of young residents in Phnom Penh, Cambodia. Habitat International, 64, 98-108 (2017).

18. Yan Zhang,Jiuchang Wei,Wei Qi.Research on the Social and Psychological Impact Model and Governance Mechanism of Emergencies__Based on the Integrated Analysis of Virtual Risk Experience and Risk Society Amplification Theory. China Emergency Management, (06):34-38 (2011).

19. Junxiu Wang,etc.Epidemic prevention and control andchanges in social mentality. Beijing: Institute of Sociology, Chinese Academy of Social Sciences.2020:1-30.

20. Peter H Lindsay,Donald A Norman.Human information Processing.An introduction to psychology[M].New York.Academic Press, 1977.

21. Min Zhang,Xuerui Liu,Yan Zhang.Analysis on the formation mechanism of audience emotion reversal of social public opinion in major disease online rescue events[J].Journal of Information,37(11):122-130 (2018).

22. Junxiu Wang,etc.The 18-day evolution of social mentality under the epidemic[R].Beijing: Institute of Sociology,Chinese Academy of Social Sciences :2-18 (2020). 